\title{
Research Briefs
}

\section{GENOMICS}

\section{Comprehensive mapping of African genetic variation}

Working with 41 co-researchers (including scientists from the Universities of the Witwatersrand and KwaZulu-Natal), Deepti Gurdasani of the African Genome Variation Project and his colleagues have produced what they believe to be the most comprehensive map of African genetic variation available to date. The significance of their work lies in the possibilities that the mapping offers for understanding the currently unknown roles that genes play in diseases such as malaria, haemorrhagic fever, Lassa fever and hypertension in sub-Saharan African populations. The team gathered data from more than 1800 people, including 320 whole genome sequences from seven populations. In the seven sequenced populations, they found 30 million genetic variants, $25 \%$ of which had never previously been identified in any human population. In their conclusion, the authors note:

A critical next step will be the large-scale deep sequencing of multiple and diverse populations across Africa, which should be integrated with ancient DNA data. This would enable the Project to identify and understand signals of ancient admixture, patterns of historical population movements, and to provide a comprehensive resource for medical genomic studies in Africa.

Gurdasani D, Carstensen T, Tekola-Ayele F, Pagani L, Tachmazidou I, Hatzikotoulas K, et al. The African Genome Variation Project shapes medical genetics in Africa. Nature. In press 2014 [published online 2014 Dec 03]. http://dx.doi.org/10.1038/nature13997

\section{PHYSICS}

\section{New results in fundamental magnetism}

André Strydom, a professor of physics and leader of the Group on Highly Correlated Matter in the Faculty of Science at the University of Johannesburg, initiated a project that has produced fascinating new results in fundamental magnetism. The work is recognised to have opened a new research field and was published on 19 November 2014 in Physical Review Letters - the flagship journal of the American Physical Society.

The work is a continuation of a project which Strydom started in 2008 on 1:2:10 structured rare-earth based ternary intermetallic compounds. His original 2009 paper is listed as the 5-year most highly cited work of the Elsevier journal Physica B: Condensed Matter (http://www.journals.elsevier. com/physica-b-condensed-matter/most-cited-articles/).

The most recent chapter in this research is a sequel to the PhD studies undertaken by P. Peratheepan, a former student of Strydom's and now Head of the Physics Department at the Eastern University of Sri Lanka. As a project which profited from a comprehensive investigative approach through joint research and international team work, the published work partners the University of Johannesburg with research institutes of the highest calibre in Germany, Taiwan and Korea.

In the study, a fascinating and unexpected interplay between electrons of two different energies and quantum mechanical states has been discovered. Traditionally, electrons pervade a metal freely in a manner described as itinerant or delocalised. This microscopic behaviour of electrons is responsible for macroscopic attributes of metals such as good electrical and thermal conductivity and metallic lustre. In the element iron, electrons occupying the spatially extended $3 \mathrm{~d}$ atomic shell are responsible for its magnetic properties. By contrast, in chemical elements known as the lanthanide elements, the magnetic electrons are found in the semi-filled $4 \mathrm{f}$ shell. These are confined to a volume much closer to the nucleus and this type of electron produces, by contrast, the localised form of magnetism. The border between local and itinerant magnetism has become a source of intense activity in condensed matter physics as a result of an astounding variety of new physical properties and unpredictable elementary particle behaviour found at this juncture. Announcements of new discoveries and exotic phenomena continue unabatedly in this topic. The discovery accompanying this paper questions some of our most basic theories of metals.

In studies that Strydom conducted with collaborators at the Max Planck Institute in Dresden during 2012/2013, surprising behaviour was found at the confluence where wave functions of these two types of electrons come into contact with each other in the same metal. An extraordinary temperaturedriven evolution among the two opposing kinds of magnetism was found. But, says Strydom:

Understanding the entangled behaviour proved to be very difficult and demanded a multi-parameter approach. Most unexpected in our studies was the transporting of one type of magnetism, namely the local-moment magnetism on the ytterbium ions with spin- and orbital forms of magnetism, into the spin-only type of magnetism on the Fe atoms. At very low temperatures, we found hints that a highly correlated state develops among the $3 d$ electrons of Fe. This state develops spontaneously when Coulomb repulsion energy among quasi-localized electrons exceed their thermal kinetic energy. In our surveys of the very low temperature properties of matter we came across collective behaviour, and a way in which the electrons seem to act in union but in ways which defy some of our most well-founded concepts of metals.

This strongly correlated state among $3 \mathrm{~d}$ electrons is highly unusual and an emergent field of condensed matter physics. The work is expected to stimulate new directions in research that will explore how different types of atomic magnetism can work together, or compete.

Khuntia P, Peratheepan P, Strydom AM, Utsumi Y, Ko K-T, Tsuei K-D, et al. Contiguous 3d and 4f magnetism: Strongly correlated 3d electrons in YbFe2Al10. Phys Rev Lett. 2014;113:216403 [published online 2014 Nov 19]. http://dx.doi.org/10.1103/PhysRevLett.113.216403

HOW TO CITE: Research briefs. S Afr J Sci. 2015;111(1/2), Art. \#a0095, 1 page. http://dx.doi.org/10.17159/sajs.2015/a0095 